\title{
CONF-860.741--7
}

NASTER

Invited paper presented at the

International Symposium on

WEAK AND ELECTROMAGNETIC INTERACTIONS IN NUCLEI

Max-Planck-Institut für Kernphysik, Heidelberg (1-5 July 1986)

\section{GLOBAL SET OF QUADRUPOLE DEFORMATION PARAMETERS FOR EVEN-EVEN NUCLEI*}

CONF-860741--7

DE86 012625

S. Raman and C. W. Nestor, Jr.

Oak Ridge National Laboratory, Oak Ridge, Tennessee 37831

\section{DISCLAIMER}

\begin{abstract}
This report was prepared as an account of work sponsored by an agency of the United States Government. Neither the United States Government nor any agency thereof, nor any of their employees, makes any warranty, express or implied, or assumes any legal liability or responsibility for the accuracy, completeness, or usefulness of any information, apparatus, product, or process disclosed, or represents that its use would not infringe privately owned rights. Reference herein to any specific commercial product, process, or service by trade trame, trademark, manufacturer, or otherwise does not necessarily constitute or imply its endorsement, recommendation, or favoring by the United States Government or any agency thereof. The views and opinions of authors expressed herein do not necessarily state or reflect those of the United States Government or any agency thereof.
\end{abstract}

"Research sponsored by the Division of Nuclear Physics, Office of High Energy and Nuclear Physics, U.S. Department of Energy, under Contract No. DE-AC05-84OR21400 with the Martin Marietta Energy Systems, Inc. 


\title{
Global Set of Quadrupole Deformation Parameters for Even-Even Nuclei
}

S. Raman and C. W. Nestor, Jr.

Oak Ridge National Laboratory, Oak Ridge, Tennessee 37831, USA

\begin{abstract}
We have completed a compilation of experimental results for the reduced electric quadrupole transition probability $[B(E 2) \dagger]$ between the $0^{+}$ground state and the first $2^{+}$state in even-even nuclei. This compilation together with certain simple relationships noted by other authors can be used to make reasonable predictions of unmeasured $B(E 2) \uparrow$ values. The quadrupole deformation parameter $\beta_{2}$ immediately follows, because $\beta_{2}$ is proportional to $[B(E 2) \dagger]^{1 / 2}$.
\end{abstract}

We have collected experimental results for the reduced electric-quadrupcle transition probability, $B(E 2) \uparrow$, between the $0^{+}$ground state and the first $2^{+}$state in eveneven nuclides [1]. These $B(E 2) \uparrow$ values represent rather basic nuclear information complementary to our knowledge of the energies of low-lying levels in these nuclides. Generally larger than expected from the single-particle model, they have emphasized the widespread occurrence of quadrupole distortions in nuclides.

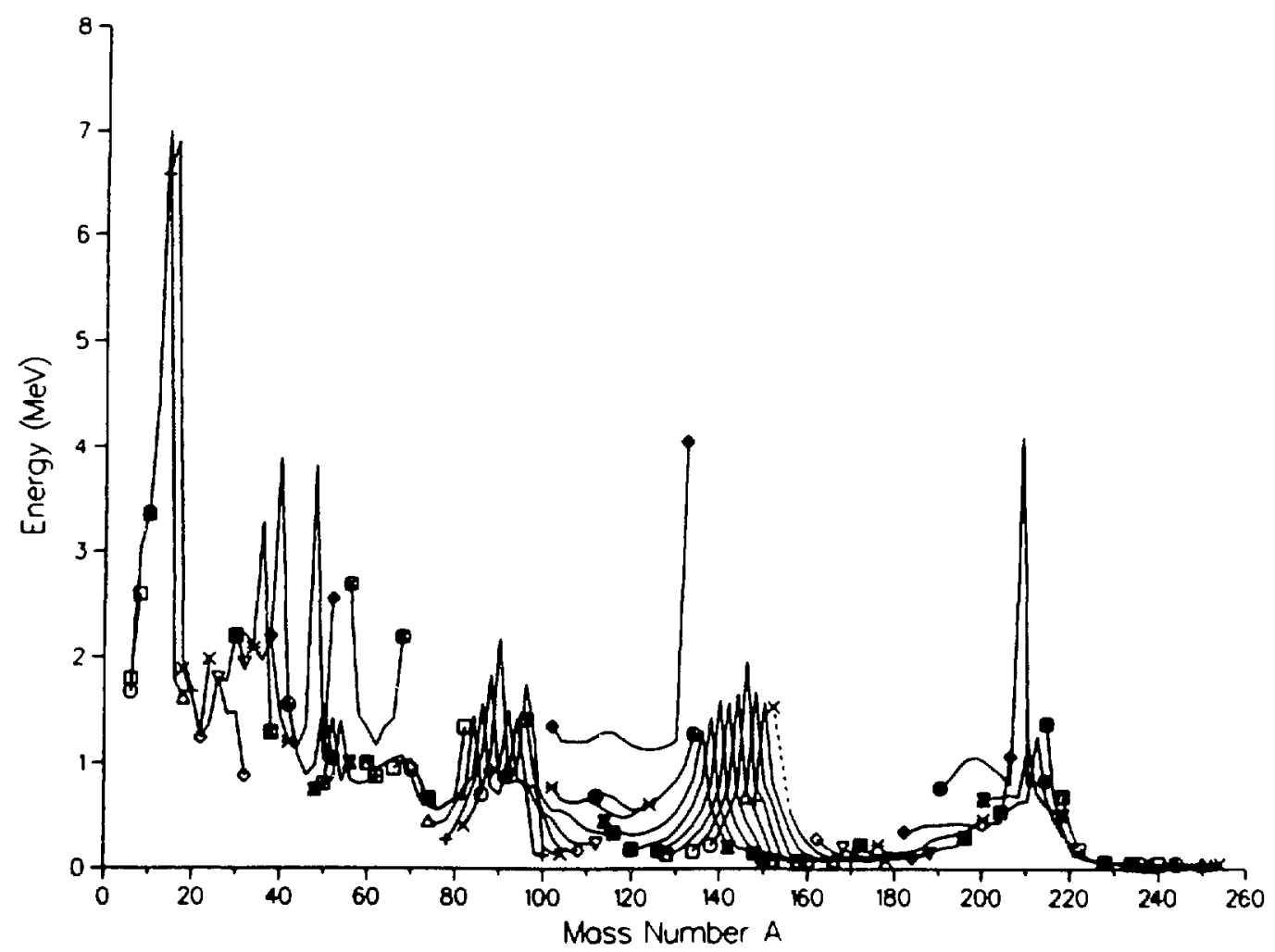

Fig. 1. Energy of the first-excited $2^{+}$state. 
Our starting point was a previous $B(E 2) \uparrow$ compilation [2], which contained 476 measured $B(E 2) \uparrow$ values from 133 references leading to adopted $B(E 2) \uparrow$ values for 155 nuclides. The current compilation contains 1765 entries from 793 references, leading to adopted $B(E 2) \uparrow$ values for 281 nuclides. The energies of the first $2^{+}$states are now known for 457 nuclides. An overall view of the data in this compilation is shown graphically in Figs. 1 and 2.

Assuming a uniform charge distribution out to the distance $R(\theta, \phi)$ and zero charge beyond, the quadrupole deformation parameter $\beta_{2}$ is related to $B(E 2)$ by the formula

$$
\beta_{2}=\left(4 \pi / 3 Z R_{0}^{2}\right)\left[B(E 2) \uparrow / e^{2}\right]^{1 / 2} .
$$

We have taken $R_{0}$ to be $1.2 A^{1 / 3}$ fermis and the single-particle $\beta_{2(\mathrm{sp})}$ to be $1.59 / Z$.

For nuclides without an experimentally determined $B\left(E^{2}\right) \uparrow$ value, a reasonable prediction based on systematics can be obtained by adopting the approaches suggested by GRODZINS [3]; HAMAMOTO [4]; ROSS and BHADURI [5]; PATNAIK, PATRA, and SATPATHY [6]; and CASTEN [7]. We have first tested these approaches with the data in our compilation. In general, predictions of $B(E 2) \uparrow$ values for nuclei off the stability line can then be made to an accuracy of $\pm 25 \%$. Such predictions can be used to deduce systematic trends in deformation as was done recently [8] in the case of the $\mathrm{Ce}, \mathrm{Nd}$, $\mathrm{Sm}$, and Gd isotopes.

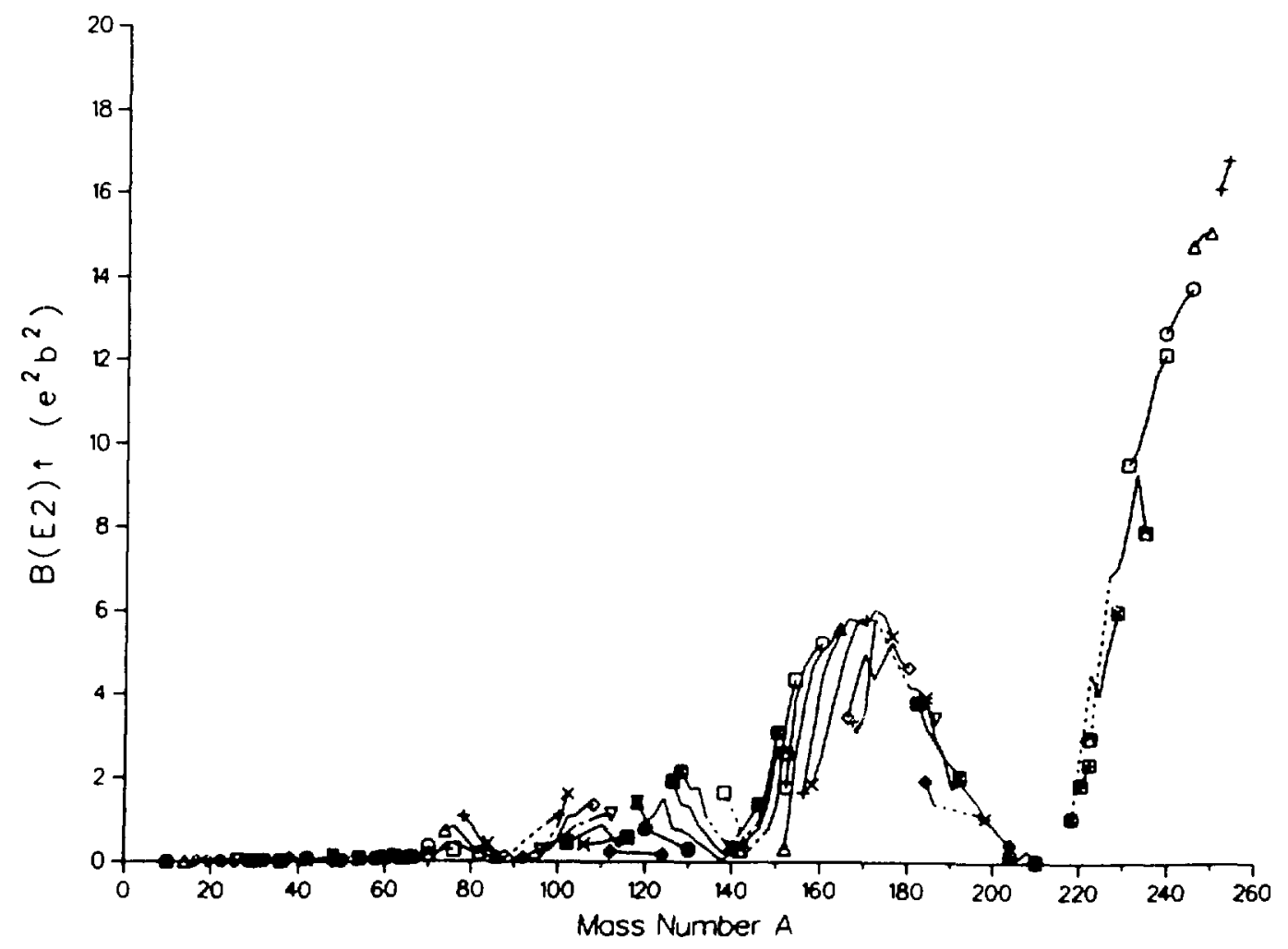

Fig. 2. Reduced transition probability, $B(E 2) \uparrow$. 
GRODZINS [3] noted first that the $\gamma$-ray mean life $\tau_{\gamma}$ was approximately proportional to $E^{-4}$, where $E$ is the energy of the $2^{+} \rightarrow 0^{+}$transition. He then suggested the following empirical relationship ( $\tau_{\gamma}$ in psec and $E$ in $\mathrm{keV}$ ):

$$
\tau_{\gamma} \approx 3.33 \times 10^{13} E^{-4} Z^{-2} A
$$

Our equivalent plot is shown in Fig. 3. A least-squares fit to the current data yielus

$$
\tau_{\gamma} \approx 5.96 \times 10^{10} E^{-3.6} Z^{-8.4} A^{7.1}
$$

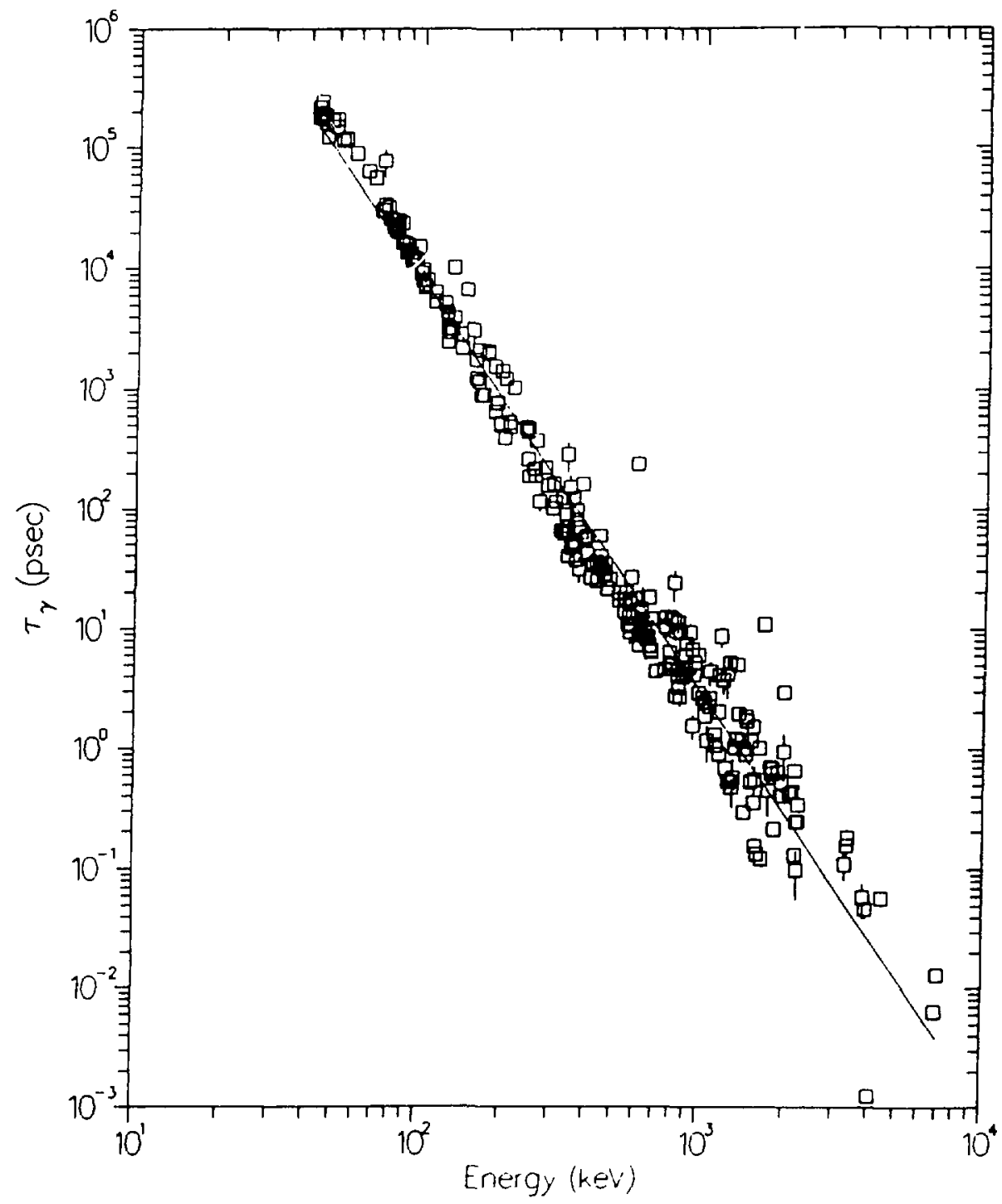

Fig. 3. Gamma-ray mean life as a function of $E$. This piot is similar to Fig. 2 of Ref. [2]. 
HАMАMOTо [4] plotted the quantity $\left[\beta_{2} / \beta_{2(\mathrm{sp})}\right] / N_{p} N_{n}$ as a function of $Z$, where the valence number of protons (neutrons) $N_{p}\left(N_{n}\right)$ is defined as the number of particles below midsheil and the number of holes past midshell. She noted that the above quantity was roughly constant over the entire mass region (both spherical and deformed) except for the regions near closed shells. Our equivalent plot is shown in Fig. 4.

After defining $F(N, Z)=[E \times B(E 2)]^{-1}$, ROSS and BHADURI [5] found that the value of $F(N, Z)$ for the anchor $N, Z$ nucleus is related to the values for the three neighboring nuclides by the difference equation

$$
F(N, Z)+F(N+2, Z+2)-F(N+2, Z)-F(N, Z+2) \sim 0 .
$$

PATNAIK, PATRA, and SATPATHY [6] further noted that a similar difference equation is also satisfied by $E$, the energy of the first $2^{+}$state, and by the reduced transition probability $B(E 2) \uparrow$.

$$
\begin{gathered}
E(N, Z)+E(N+2, Z+2)-E(N+2, Z)-E(N, Z+2) \sim 0 \\
B(E 2)[N, Z]+B(E 2)[N+2, Z+2]-B(E 2)[N+2, Z]-B(E 2)[N, Z+2] \sim 0 .
\end{gathered}
$$

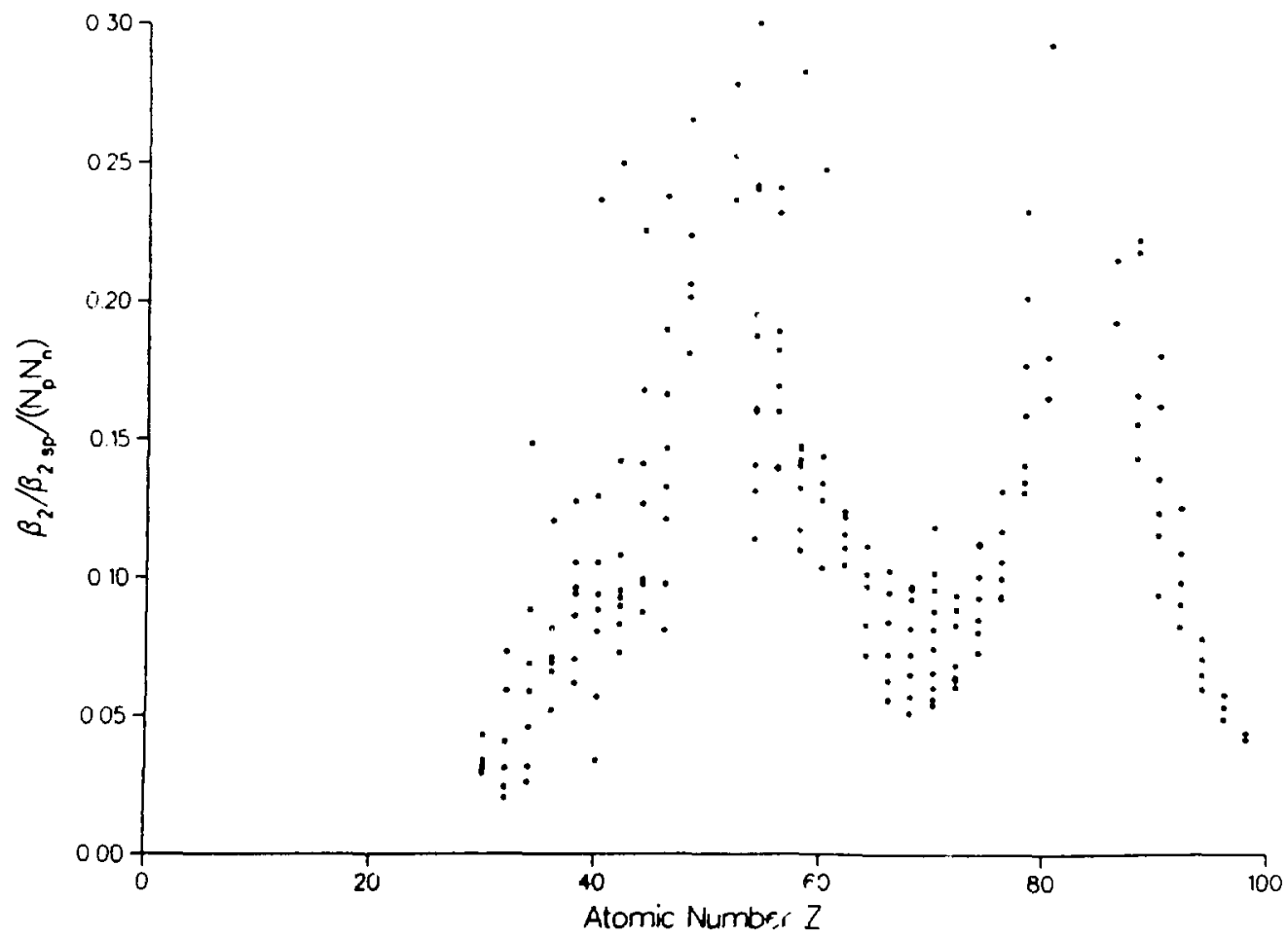

Fig. 4. Quadrupole deformation parameter, $\beta_{2}$ divided by $\beta_{2(\mathrm{op})}(=1.59 / Z)$ as a function of $Z$. This plot is similar to Fig. 2 of Ref. [4]. 
We have tested the difference equations (4) and (6) with the data in our compilation. The results are shown in Fig. 5. Except near closed shells, both approaches yield deviations of the difference equations from zero that are typically $\pm 25 \%$. Even though they were proposed more than a decade ago, these difference equations have not received the attention that they probably deserve.
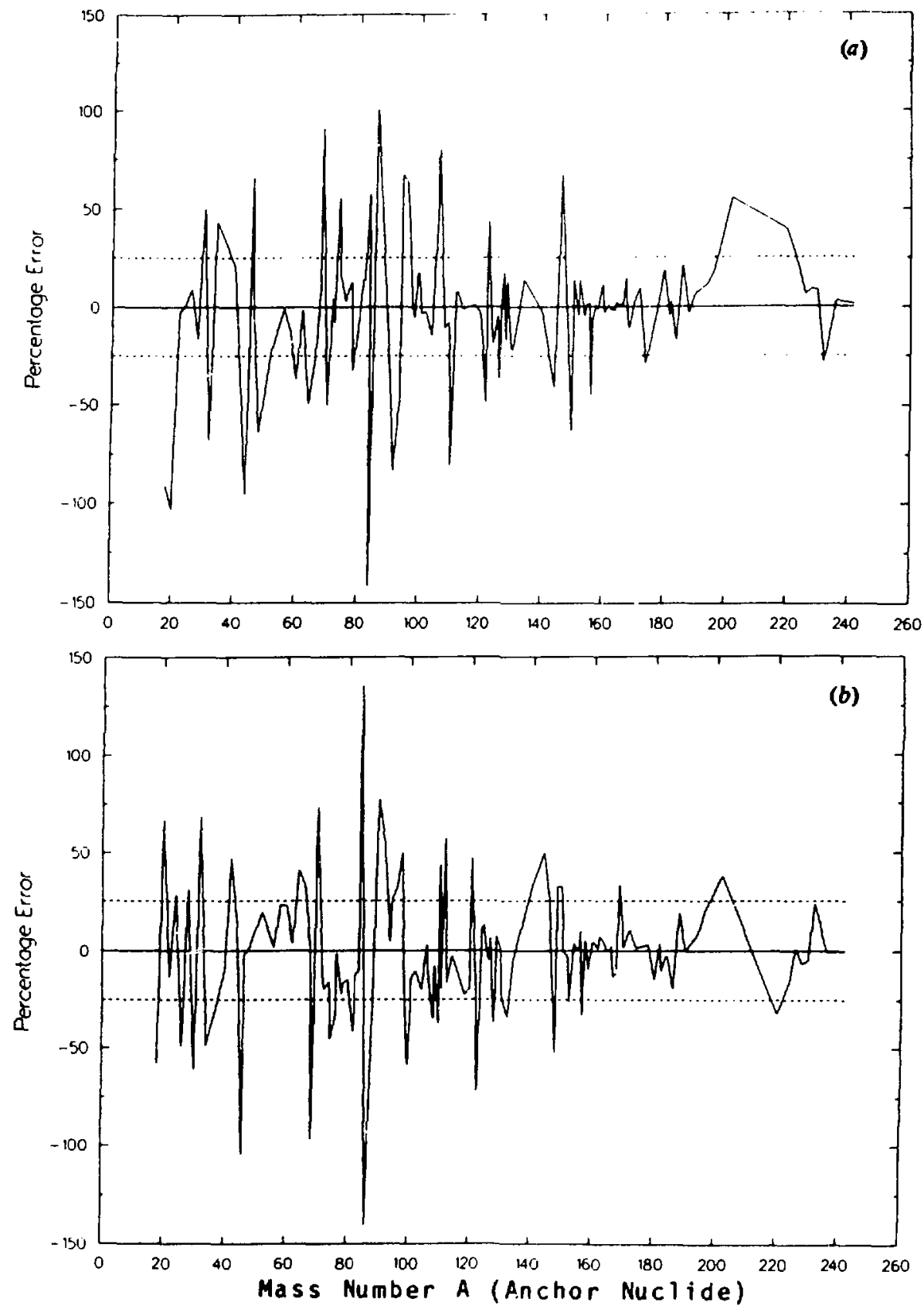

Fig. 5. Test of the difference equations of (a) ROsS and bHADURI [5]-see Eq. (3), and (b) PATNAIK, PATRA, and SATPATHY [6] - see Eq. (5). The deviation from zero of either Eq. (3) or Eq. (5) is divided by the average of the four values, expressed as a percentage, and plotted along the ordinate. 
Finally, CASTEN [7] has recently attempted a systemization of a considerable amount of spectroscopic data, especially in the five nuclear transition regions $[A \approx$ $100, A \approx 130, \mathrm{~A} \approx 150(\mathrm{Z}<64), A \approx 150(Z \geqslant 64)$, and $A \approx 190]$ in terms of the valence nucleon product $N_{\rho} N_{n}$. He has shown that the $B(E 2)$ values in the $A \approx 150$ (Z $\leqslant 64), A \approx 150(Z \geqslant 66)$, and $A \approx 100$ regions fall on relatively smooth curves. Some of CASTEN's conclusions are based on a posteriori counting of valence nucleons. Separating the $A \approx 150$ region into $Z \leqslant 64$ and $Z \geqslant 66$ parts is just one example, for which he has provided ample justification in terms of a subshell closure. Our equivalent plot for the $A \approx 100$ region is shown in Fig. 6. We use his prescription that if $N<60$, the proton shell is 38 to 50 , and if $N \geqslant 60$, the proton shell is 28 to 50 .

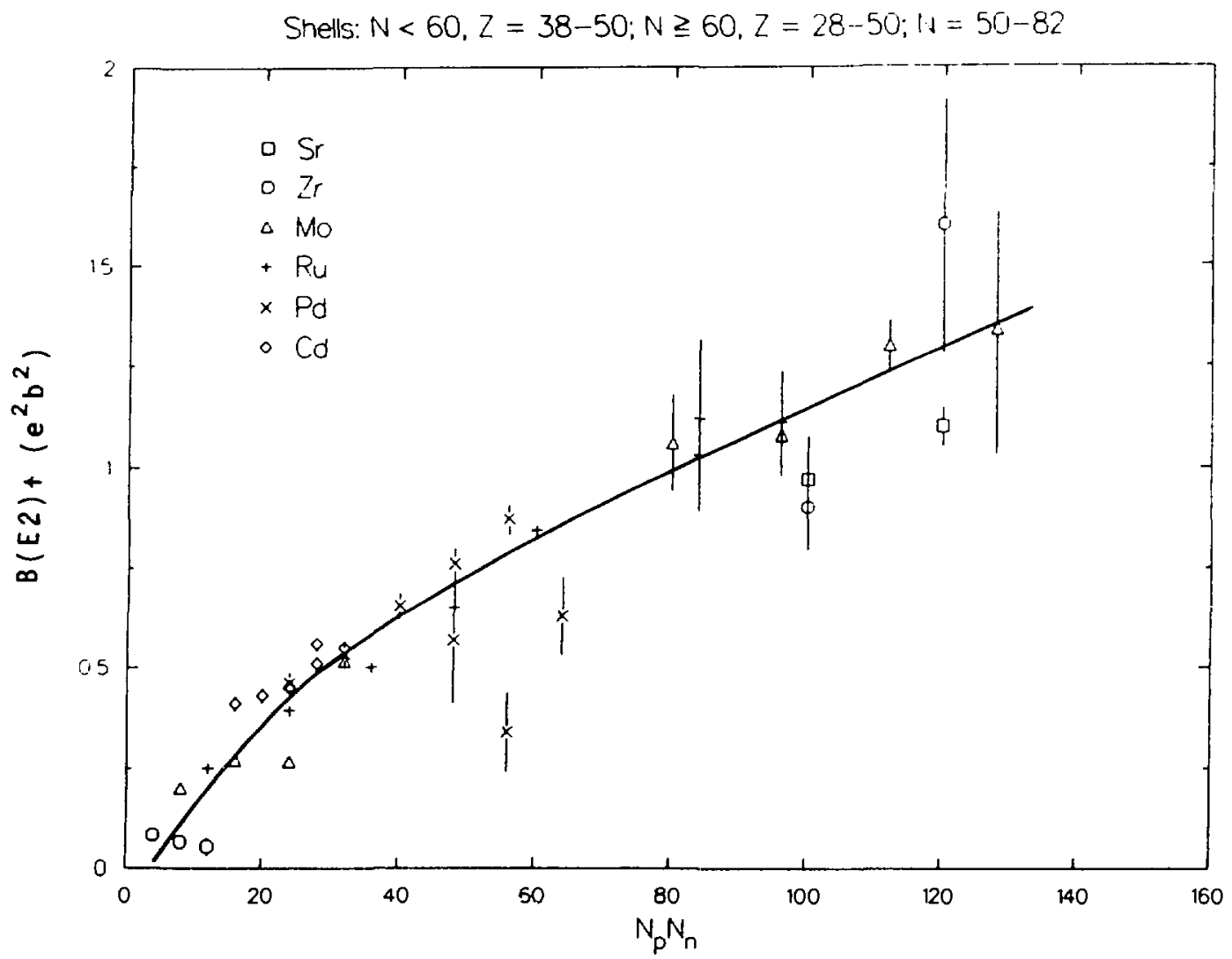

Fig. 6. $B(E 2)$ systematics for the $A \approx 100$ region. This plot is similar to Fig. 25 of Ref. [7]. The curve is drawn to guide the eye.

Retvrning to HAMAMoto's [4] original idea, we have plotted in Fig. 7 the quantity $\beta_{2} / \beta_{2(\text { sp) }}$ vs $N_{p} N_{n}$ for the isotopes of eight even- $Z$ elements with $64<\mathrm{Z}<82$. The curve drawn there accounts rather well for 61 data points.

We have shown that there are several ways of systematizing the $B(E 2)$ values

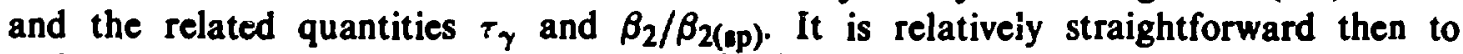
arrive at a global set of recommended $B(E 2)$ and $\beta_{2}$ values by combining measured values with predictions. We are currently in the process of arriving at such a set. 
Shells: $Z=50-82 ; N=82-126$

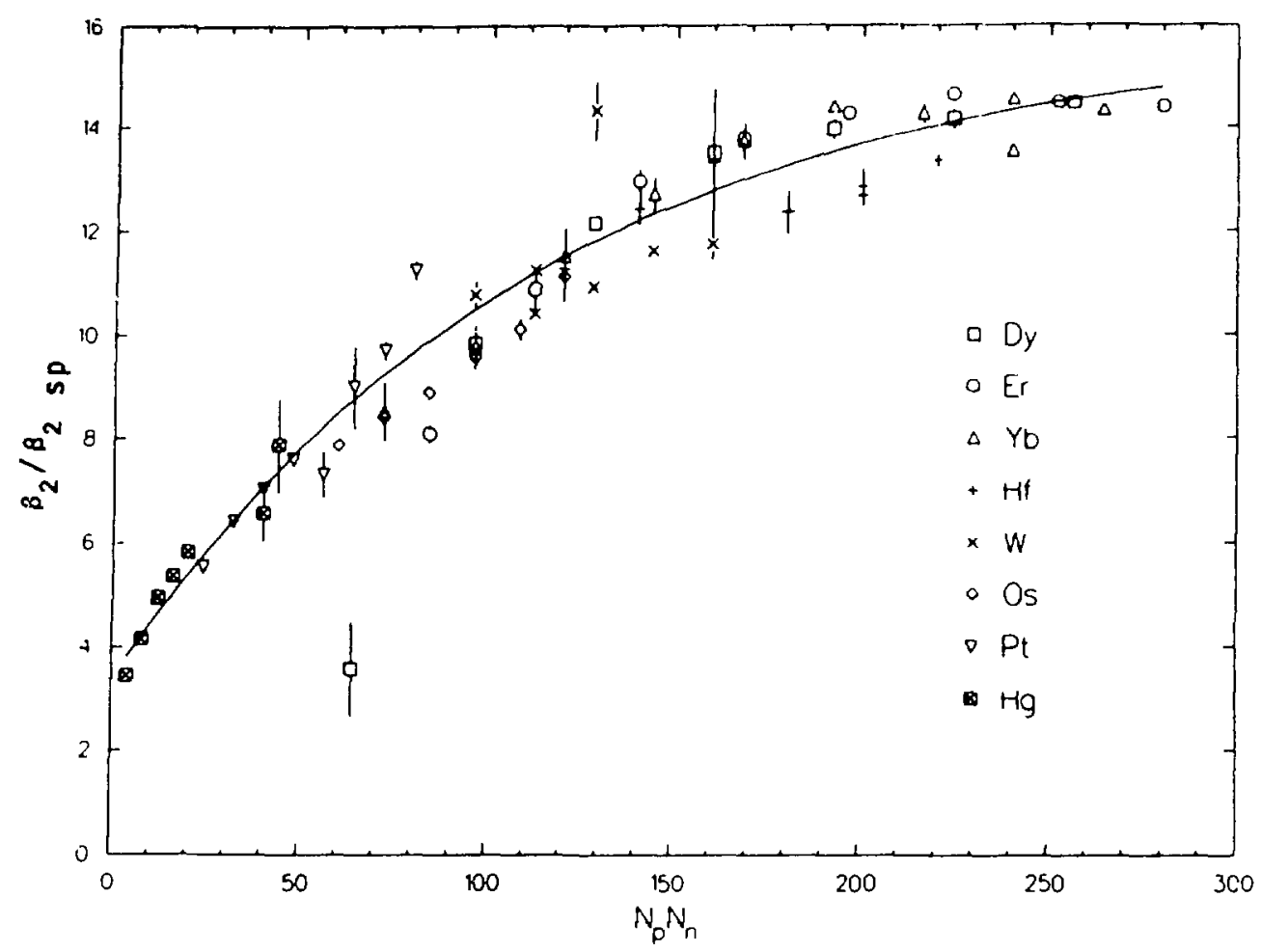

Fig. 7. Quadrupole deformation parameter, $\beta_{2}$, divided by $\beta_{2(\mathrm{sp})}(=1.59 / Z)$ as a function of the quantity $N_{p} N_{n}$ for even- $Z$ isotopes in the $64<Z<82$ region. The curve is drawn to guide the eye.

This research was sponsored by the Division of Nuclear Physics, Office of High Energy and Nurlear Physics, U.S. Department of Energy, under Contract No. DEAC05-84OR2146 with the Martin Marietta Energy Systems, Inc.

\section{References}

[1] S. Raman, C. H. Malarkey, W. T. Milner, C. W. Nestor, Jr., and P. H. Stelson, At. Data Nucl. Data Tables, to be published.

[2] P. H. Stelson and L. Grodzins, Nucl. Data A1, 21 (1965).

[3] L. Grodzins, Phys. Lett. 2, 88 (1962).

[4] I. Hamamoto, Nucl. Phys. 73, 225 (1965).

[5] C. K. Ross and R. K. Bhaduri, Nucl. Phys. A196, 369 (1972).

[6] R. Patnaik, R. Patra, and L. Satpathy, Phys. Rev. C 12, 2038 (1975).

[7] R. F. Casten, Nucl. Phys. A443, 1 (1985).

[8] C. J. Lister et al., Phys. Rev. Lett. 55. 810 (1985). 